\title{
Editorial
}

\section{Just How Critical is Epithelial-to-Mesenchymal Transition (EMT) to Metastatic Dissemination?}

\author{
Torre-Healy $\mathrm{LA}^{1,2}$ and Chia-Hsin Chan ${ }^{1,3^{*}}$ \\ ${ }^{1}$ Department of Pharmacological Sciences, Stony Brook University, Stony Brook, NY 11794, USA \\ ${ }^{2}$ Medical Scientist Training Program, Stony Brook University, Stony Brook, NY, 11794, USA \\ ${ }^{3}$ Stony Brook Medicine Cancer Center, Stony Brook University, Stony Brook, NY 11794, USA \\ *Corresponding author: Chia-Hsin Chan, Department of Pharmacological Sciences, Stony Brook University, Stony \\ Brook, NY 11794-8651, USA, Tel: +1 6314443085; E-mail: chia-hsin.chan@stonybrook.edu
}

Received Date: August 03, 2017; Accepted Date: August 04, 2017; Published Date: August 24, 2017

Epithelial-to-Mesenchymal Transition (EMT) has long been considered to be critical for the development of lung metastasis in breast cancer [1]. Cells that transition to a mesenchymal phenotype lose their adherent properties and become increasingly motile. Silencing of Snail, Zeb1, Twist and other EMT associated genes correlate with a decrease in metastasis in in vivo models, and point to a critical role of EMT in metastasis [2-4]. However, analysis of tumor cells within both the primary tumor and metastatic lesions show primarily epithelial markers. Currently, it is believed that cells undergo Mesenchymal-toEpithelial Transition (MET) following their colonization into a new organ environment [5]. A recent publication by Fischer et al., used the Cre/CreER lineage-tracing genetic mouse model to explore the necessity of EMT in breast cancer metastasis [6]. Through their generation of Fibroblast Specific Protein 1 (FSP1)-GFP and Vimentin-GFP reporter mouase lines, using Cre and Cre-ER respectively, they reported that cancer cells that had not undergone EMT still metastasized just as much as those that had. The conclusions of this paper were brought into question in 2017 by Ye et al., following close scrutiny of selection of Vimentin and FSP1 promoters for the activation of EMT programs as well as the recombination efficiency of CreER [7]. We will discuss the positions held by the two contradictory publications and provide insight beyond luminal-subtype breast cancer.

Fischer et al., created two oncogene-driven triple-transgenic mouse models, MMTV-PyMT/Rosa26-RFP-GFP/FSP1-Cre (Tri-PyMT) and MMTV-neu/Rosa26-RFP-GFP/FSP1-Cre, (Tri-Neu) by crossing MMTV-PyMT or MMTV-neu mice with Cre-switchable fluorescent reporter (ROSA26-lox-RFP-STOPlox-GFP) and FSP1-Cre mice to track EMT during metastasis in vivo. Their assumption was that the EMT process will switch fluorescence expression from RFP to GFP. Thus, RFP and GFP would serve as readout to monitor cells in epithelial and mesenchymal state, respectively. Unexpectedly, Fischer et al., showed that the metastatic lesions in the lungs mostly consist of $\mathrm{RFP}^{+}$cells. Following this observation, they attempted to inhibit EMT process by ectopic expression of miR-200, an endogenous inhibitor of Zeb1. Overexpression of miR-200 resulted in the disappearance of the $\mathrm{GFP}^{+}$population, with $>90 \%$ of cells retaining their RFP expression. However, it also had no effect on the efficiency of metastasis in animal models. This led them to claim that EMT has a negligible contribution to metastasis in breast cancer.

The data form Fischer et al., have stimulated provocative discussions. Ye et al., published a rebuttal two year following that scrutinized the data at a deeper level [7]. They found a number of concerns with the validity of the claims. The variety of methods for EMT program activation requires the careful selection of an appropriate marker within a given tissue system. Ye et al., showed that either FSP1 or Vimentin alone were insufficient drivers of EMT. In their own hands, Vimentin was coexpressed with Snail in only $25 \%$ of cells, while it was coexpressed with Zeb1 in $60 \%$. FSP1 on the other hand, only coexpressed with either Snail or Zeb1 in approximately $10 \%$ of cells. The observations that FSP1 or Vimentin were present in a small fraction of carcinoma cells that had possessed active EMT program, indicated by the expression of Snail or Zeb1 suggest FSP1 and Vimentin-mediated fluorescence switch may not best manifest the EMT activation. Moreover, a number of Vimentin ${ }^{+}$cells were found to exhibit negative GFP expression, implying the incompleteness of the Cre-driven recombination. Taken together, these data prohibit the extrapolation of Vimentin and FSP1 mediated reporter systems to EMT process as a whole. They next looked at the validity of miR-200 as an inhibitor of EMT. Several studies have shown 
Citation: Torre-Healy LA, Chia-Hsin Chan (2017) Just How Critical is Epithelial-to-Mesenchymal Transition (EM) to Metastatic Dissemination? J Pharmacol clin Trials 2017: 8-10. DOI: https://doi.org/10.29199/JPCT.101015.

that miR-200 also exhibits off target effects on Sec23, which has pro-metastatic events [8]. A decrease in Zeb1 is to be expected, but the effects on Sec23 are sufficient to offset the decrease in EMT. This accounts for the complete eradication of GFP cells without the abrogation of metastasis. Altogether, these conflicting observations cast the power of Fischer et al.'s experiments and claims into debate.

Despite the debate, Fischer's work lays the important foundation for further interrogation of EMT with the utilization of lineage tracing systems. Coupling the GFP reporter system with more powerful driver genes, such as Snail or Zeb1, could prove to be an exciting method for deducing the importance of EMT in metastasis. It also highlights the need for more thorough analysis of the interplay between the variety of EMT programs and which markers should be relied upon in each tissue system.

Breast cancer is a heterogeneous group of diseases that consists of at least five subtypes with distinct morphological features and clinical behaviors: luminal A, luminal B, normal breast-like , Human Epidermal growth factor Receptor 2 (HER2) and basal-like [9]. Luminal A, Luminal B and HER2-enriched tumors retain most epithelial features, while basal-like tumors exhibit both basal and mesenchymal features. Gene expression profiling and experimental studies have showed that basal-like breast cancer has the strongest association with EMT program [10,11]. It would be interesting and informative to employ the lineage tracing system to study the influence of EMT in basal-like subtype mouse models.

\section{Acknowledgement}

C. H. C was supported by the TRO Carol M. Baldwin Award, the NYS Peter T. Rowley Breast Cancer Scientific Research Project and NIH grants (K22 CA181412 and R01CA211912). L. A. T-H was supported by NIH T32 GM008444

\section{References}

1. Nieto MA, Huang RY, Jackson RA, Thiery JP (2016) EMT: 2016. Cell 166: 21-45.

2. Ni T, Li XY, Lu N, An T, Liu ZP, et al. (2016) Snail1-dependent p53 repression regulates expansion and activity of tumour-initiating cells in breast cancer. Nat Cell Biol 18: 1221-1232.

3. Lee HJ, Li CF, Ruan D, Powers S, Thompson PA, et al. (2016) The DNA Damage Transducer RNF8 Facilitates Cancer Chemoresistance and Progression through Twist Activation. Mol Cell 63: 1021-1033.

4. Tran HD, Luitel K, Kim M, Zhang K, Longmore GD, et al. (2014) Transient SNAIL1 expression is necessary for metastatic competence in breast cancer. Cancer Res 74: 6330-6340.

5. Ye X, Weinberg RA (2015) Epithelial-Mesenchymal Plasticity: A Central Regulator of Cancer Progression. Trends Cell Biol 25: 675686.

6. Fischer KR, Durrans A, Lee S, Sheng J, Li F, et al. (2015) Epithelialto-mesenchymal transition is not required for lung metastasis but contributes to chemoresistance. Nature 527: 472-476.

7. Ye X, Brabletz T, Kang Y, Longmore GD, Nieto MA, et al. (2017) Upholding a role for EMT in breast cancer metastasis. Nature 547: 1-3.

8. Korpal M, Ell BJ, Buffa FM, Ibrahim T, Blanco MA, et al. (2011) Direct targeting of Sec23a by miR-200s influences cancer cell secretome and promotes metastatic colonization. Nat Med 17: 1101-1108.

9. Vargo-Gogola T, Rosen JM (2007) Modelling breast cancer: one size does not fit all. Nat Rev Cancer 7: 659-672.

10. Prat A, Perou CM (2011) Deconstructing the molecular portraits of breast cancer. Mol Oncol 5: 5-23.

11. Sarrio D, Rodriguez-Pinilla SM, Hardisson D, Cano A, Moreno-Bueno $\mathrm{G}$, et al. (2008) Epithelial-mesenchymal transition in breast cancer relates to the basal-like phenotype. Cancer Res 68: 989-997. 